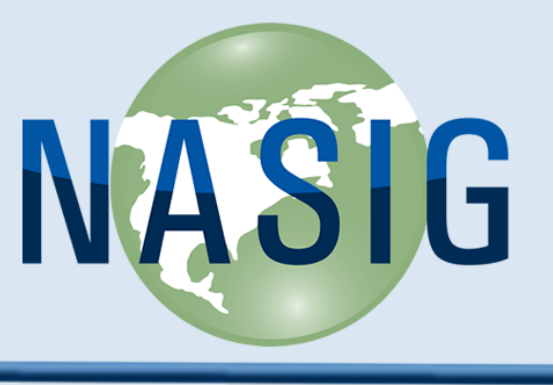

Vol. 28 , no. 4
Newsletter

December 2013
North American Serials Interest Group

eISSN 1542-3417

\title{
Discovering Music: Small-scale, Web-scale, Facets, and Beyond
}

\author{
Rebecca Belford, University at Buffalo \\ Tracey Snyder, Cornell University
}

Reported by Patrick L. Carr

Web-scale discovery tools are currently transforming the interfaces libraries provide for the discovery and access of their collections. Although these tools are significantly enhancing user experiences, they are also introducing new challenges. The concurrent session "Discovering Music: Small-scale, Web-scale, Facets, and Beyond" examined one such challenge: the unique difficulties of organizing and searching for music materials (e.g., scores, sheet music, and recordings) in a web-scale environment.

In the session's first presentation, Rebecca Belford (music cataloger/reference librarian) provided an overview of some of the specific complexities that make the discovery of music materials problematic in webscale discovery interfaces. She noted that these complexities should be of interest to anyone engaged with the challenges of library collection discovery because music materials measure how well discovery tools function at the extremes; in other words, a discovery tool that works well for music materials will also work well for most other library materials. Next, Belford discussed how the Music Library Association's Music Discovery Requirements document (http://goo.gl/FQk2U) aims to address these complexities. As she explained, this document, which was released in April 2012, provides a range of best practices and recommendations detailing the characteristics of music materials and providing 1 guidance regarding how the administrators of webdiscovery tools can harness AACR2 and RDA standards within the MARC record format to maximize the discoverability of music materials. The document has a FRBR-like structure, and Belford's presentation devoted particular attention to discussing the ramifications of music formats and works within the discovery context, including the navigation between different manifestations of the same work. Finally, Belford highlighted some significant developments in music discovery occurring outside of the traditional library environment, including the application of FRBR principles at the Australian Music Centre.

The session's second presenter, Tracey Snyder (assistant music librarian), considered the discoverability of music materials within the specific discovery interfaces being developed and implemented at Cornell University. After reviewing Cornell's current discovery interfaces, Snyder described the university's efforts to implement the faceted open source discovery layer, Blacklight, as their main catalog interface; Cornell aims to have a beta release accessible to patrons during the 2013/2014 academic year. Snyder is a member of the Blacklight implementation team and is playing a particular role as an advocate for the effective discovery of music materials. She worked with patrons to conduct usability testing for music materials, and, in doing so, she was able to identify strengths and weaknesses NASIG Newsletter

December 2013 
related to the discovery of music materials via the

Blacklight interface. Collaborating with other members of the implementation team, she was able to address certain problems identified in the usability testing, but resolutions to other problems are still in progress.

Snyder concluded by noting some directions for the future development of music discoverability, including work by the Library of Congress, in cooperation with the Music Library Association, incorporating RDA elements in order to achieve more granularity in search results and creating thesauri for genre/form and medium of performance. 